\title{
Rotor Dynamical Characteristics of Helicopter Start
}

\author{
Hongru Ma, Yue Wang, Zhitao Mu, Wensheng Sun \\ Department of Aeronautical Mechanism, Naval Aeronautical and Astronautical University Qingdao \\ Branch, Qingdao 266041, China
}

1866483895@163.com,swszcl@163.com,mzt63@126.com

Keywords: Dynamics of rotor, flap, response

\begin{abstract}
Based on the theory of finite rotation beam, together with the finite element method, the rotor dynamic model is established, and the relationship of strain and displacement in the strain energy of blade is solved by introducing the generalized Danielson-Hodges strain and Green-Lagrange strain. The three-dimensional nonlinear analysis is decoupled into two-dimensional linear profile analysis and one-dimensional nonlinear blade analysis. Hartenberg-Denarit augmented transformation matrix is introduced in kinetic modeling. According to the Hamilton principle, the nonlinear implicit dynamic equations of the rotor are established. Blade flap under the influence of gust wind is calculated; the safety criterion of the ground starting of the helicopter is put forward.
\end{abstract}

\section{Introduction}

In the United States, there has been many accidents of such collision in the process of starting or stopping of the blades, which is called 'Strike Tunnel'. In order to avoid the occurrence of such accidents, this paper studies the flap response characteristics of the blades in the starting process of helicopter using the rotor starting model built, and provides a safe basis for the starting operation of the helicopter.

Different from the traditional isotropic dynamics model which is based on the moderate deflection beam theory, this paper adopts the finite rotation beam theory to deal with the elastic deformation of blade, and sets up an accurate model of rotor dynamics with the method of finite element. The paper also discusses the characteristics of composite blade, the key structural design parameters (pre cone angle, torsion angle and sweep angle etc. of blade, the characteristics of typical hub configuration, and the coupling motion and nonlinear deformation of flap, shimmy and torsion in the collision. The definition of generalized Danielson-Hodges strain is introduced in strain energy modeling, and the three-dimensional nonlinear analysis of composite blade is decoupled into two-dimensional linear profile analysis and one-dimensional nonlinear blade analysis. Meanwhile, the Hartenberg-Denarit augmented transformation matrix is introduced in kinetic modeling. This paper creates a new recursive method of identifying the position vector of any point on the blade in inertial coordinates, and finally establishes the nonlinear implicit dynamic equations of the rotor according to the Hamilton principle.

\subsection{Rotation beam}

Euler-Bernoulli beam assumption is used to model the blade. After deformation, the position coordinates of the origin in the undeformed coordinate system is

$$
R_{1}=\left(\begin{array}{lll}
0 & \eta & \xi
\end{array}\right)\left[\begin{array}{l}
i_{x}{ }^{\prime} \\
j_{n}{ }^{\prime} \\
k_{\xi}{ }^{\prime}
\end{array}\right]=S\left[\begin{array}{l}
i_{x}{ }^{\prime} \\
j_{n}{ }^{\prime} \\
k_{\xi}{ }^{\prime}
\end{array}\right]
$$

In the non-deformation coordinates, the position augmented vector of Point $\mathrm{P}$ after augmentation is

$S^{2}=\left(\begin{array}{llll}S_{x}^{2} & S_{y}^{2} & S_{z}^{2} & 1\end{array}\right)=S^{1} H^{1}$ 
$H^{1}$, also called the Hartenberg-Denarit transformation matrix, is the augmented transformation matrix in the non-deformation coordinates. It can be written as

$$
H^{1}=\left[\begin{array}{cc}
T_{1} & 0 \\
W_{1} & 1
\end{array}\right]
$$

To facilitate the computer programming, the position augmented vector and the transformation matrix can be recursively expressed as

$$
\begin{aligned}
& S^{i}=\left(\begin{array}{llll}
S_{x}^{i} & S_{y}^{i} & S_{z}^{i} & 1
\end{array}\right)=S^{i-1} H^{i-1} \\
& H^{i-1}=\left[\begin{array}{cc}
T_{i-1} & 0 \\
W_{i-1} & 1
\end{array}\right]
\end{aligned}
$$

The rest may be deduced by analogy. In inertial coordinate system, the position augmented vector of any point $(\mathrm{P})$ can be expressed as

$$
S^{n}=\left(\begin{array}{llll}
S_{x}^{n} & S_{y}^{n} & S_{z}^{n} & 1
\end{array}\right)=S^{n-1} H^{n-1}=S^{1} H^{1} \ldots H^{i} \ldots H^{n-1}
$$

The position vector of Point $\mathrm{P}$ is

$$
R_{n}=\left(\begin{array}{lll}
S_{x}^{n} & S_{y}^{n} & S_{z}^{n}
\end{array}\right)\left\{\begin{array}{l}
i_{b} \\
j_{b} \\
k_{b}
\end{array}\right\}
$$

From the above formula, it can be known that the position vector in the inertial coordinate system can be obtained by the recursive calculation, provided that the position of the origins in the coordinate systems and their conversion relationship are verified. It simplifies the calculation of rigid-flexible coupling, and improves the applicability of the algorithm for different rotor structure and motion form.

\subsection{Kinetic energy term}

Kinetic energy variation of a single blade can be written as

$$
\delta T_{b}=\iiint_{V} \frac{1}{2} \rho_{b} \delta \dot{\boldsymbol{R}}_{n}^{2} \mathrm{~d} V=\iiint_{l} \rho_{A} \ddot{\boldsymbol{R}}_{n} \sum_{i=1}^{n_{b}} \frac{\partial \boldsymbol{R}_{n}}{\partial q_{i}} \mathrm{~d} A \mathrm{~d} l \delta q_{i}
$$

With 0 time end condition introduced, $Q T i$, the Number i generalized force generated by kinetic energy can be deductively obtained. After calculating derivative, the matrixes of tangent mass, damping and stiffness, generated by kinetic energy, are respectively written as

$$
\begin{aligned}
& M_{i j}^{\mathrm{T}}=\frac{\partial Q_{i}^{\mathrm{T}}}{\partial \ddot{\boldsymbol{q}}_{j}}=\int_{l} \mathrm{~d} l \iint_{A} \rho_{b} \frac{\partial \boldsymbol{R}_{n}}{\partial q_{i}} \cdot \frac{\partial \boldsymbol{R}_{n}}{\partial q_{j}} \mathrm{~d} A \\
& C_{i j}^{\mathrm{T}}=\frac{\partial Q_{i}^{\mathrm{T}}}{\partial q_{j}}=-\int_{l} \iint_{A} 2 \rho_{b} \frac{\partial \dot{\boldsymbol{R}}_{n}}{\partial q_{j}} \cdot \frac{\partial \boldsymbol{R}_{n}}{\partial q_{i}} \mathrm{~d} A \mathrm{~d} l \\
& K_{i j}^{\mathrm{T}}=\frac{\partial Q_{i}^{\mathrm{T}}}{\partial q_{j}}=-\iint_{l} \int_{A} \rho_{b}\left(\frac{\partial \dot{\boldsymbol{R}}_{n}}{\partial q_{j}} \cdot \frac{\partial \boldsymbol{R}_{n}}{\partial q_{i}}+\ddot{\boldsymbol{R}}_{n} \cdot \frac{\partial^{2} \boldsymbol{R}_{n}}{\partial q_{i} \partial q_{j}}\right) \mathrm{d} A \mathrm{~d} l
\end{aligned}
$$

The related derivatives and partial derivatives of augmented position vector can be expressed by using the recursive algorithm, which will greatly improve the generality of derivation.

\subsection{Induced velocity}

The calculation of induced velocity adopts the improved Glauert inflow model. Inflow is connected with the pull coefficient of rotor, forward ratio and dip angle of blade. On the basis of the Glauert uniform inflow model, the improved linear inflow model further assumes that the induced inflow should have a linear distribution along the radius of blade. 


$$
\lambda_{i}=\frac{C_{T} / 2}{\sqrt{\lambda^{2}+\mu^{2}}}\left(1+\kappa_{x} x \cos \Psi+\kappa_{y} x \sin \Psi\right)
$$

\subsection{Equation of blade}

Establish the motion equations of the rotor system with application of Hamilton principle.

$$
\int_{t_{1}}^{t_{2}}\left[\delta\left(U_{b}-T_{b}\right)-\delta W_{A}\right] \mathrm{d} t=0
$$

\subsection{Solutions for Response}

In this paper, the starting response is kind of transient responses, so the problem is solved by the implicit Newmark integral method.

\section{Safety Criterion}

In the process of starting or stopping of the ship-based helicopters, rotor speed is low, so the centrifugal force generated is not powerful enough to limit the waving angle of the blade. When the blade waves down too much, there exists a danger of collision with the fuselage. Therefore, it is necessary to determine the safety standards of the distance between the blades and the body, and prevent the occurrence of the impact accident.

In view of the fact that the distance between the tip of the blade and the fuselage is the final indicator to measure the safety of starting and stopping, this paper introduces BCS (Blade Clearance Scale) of the US Navy as the standard. As shown in Table 1. If $\mathrm{BCS}=1$, it indicates that the distance between blade and fuselage is more than 23 inches $(58.42 \mathrm{~cm})$, while it means a collision between blade and fuselage when $\mathrm{BCS}=4$.

Table 1 Definition of BCS

\begin{tabular}{|c|l|}
\hline $\begin{array}{c}\text { BCS } \\
\text { Coefficient }\end{array}$ & $\begin{array}{c}\text { Distance between Blade and } \\
\text { Fuselage } \\
\text { in(cm) }\end{array}$ \\
\hline 1 & $>23(58.42)$ \\
\hline 2 & $8 \sim 23(20.32 \sim 58.42)$ \\
\hline 3 & $0 \sim 8(0 \sim 20.32)$ \\
\hline 4 & $<0$ \\
\hline
\end{tabular}

\section{Analysis of Calculation}

Taking a certain type helicopter for example, this paper calculates the motion characteristics of blade under different instantaneous wind speeds. Since the most likely scenario is the collision between blade and tail beam, the paper focuses on the calculation of the response characteristics of the blades on the tail beam.

The down-flap value of blade when the blade reaches $180^{\circ}$ azimuth. The rings from inside to outside in this figure represent the flap value (in feet); the unit of wind speed is knot. $180^{\circ}$ azimuth is located on the tail beam. Down-flap value increases gradually with the rise of transient wind speed the blade bearing. When the transient wind speed reaches 40 knots, the down-flap value of blade's tip is about 1.8 feet $(53 \mathrm{~cm})$, while the vertical distance between the rotor structure and the tail beam is about $50 \mathrm{~cm}$. The BCS coefficient is 4 at this point, there is a possibility that the blades may be struck by the tail beam. So as to ensure the safety, the helicopter can not be started under such wind speed.

Figure 1 shows the blade's flapping angle (up) and oscillating angle (down) when the speed of gust wind is 40 knots. Under the action of gust wind, the flapping angle of blade appears a large swing, and the final value is fixed. At the initial stage of starting, the blades' rotation speed is low, thus the Coriolis force makes the blades oscillate under the action of strong flap. Due to the low rotor speed, the centrifugal force is too small to produce enough oscillating restoring moment, so the blades present a big and disordered oscillating angle. As the rotor speed increases gradually, oscillating angle tends to be stabilized after the flapping angle region becomes stable. 


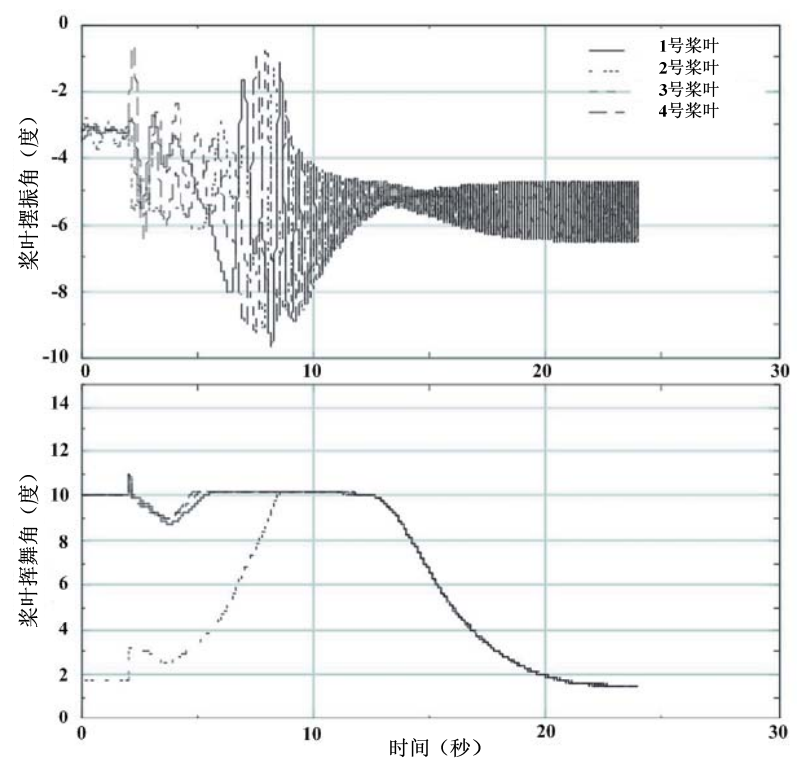

Fig. 1 Blade's Flapping Angle (up) and Oscillating Angle (down)

Under the initial action of gust wind, turbulence of a certain degree occurs on the fuselage due to an overlarge flap of blade, which is reflected in the irregular compression value of the undercarriage. As time goes on, the compression of the undercarriage tends to be stable after the blade flapping motion gets stabilized.

\section{Conclusions}

From the calculation above we know that the gust wind has a great influence on the motion characteristics of blade flapping during the process of helicopter ground starting. It is necessary to make a clear limit to the transient wind speed during the process of the helicopter's starting to prevent the blades from colliding with fuselage because of a large flap.

\section{References}

[1]. Houbolt J C, Brooks G W. Differential equations of motion for combined flapwise bending, chordwise bending, and torsion of twisted nonuniform rotor blade. NACA Report 1346, 1958.

[2]. SUN C W, The influence of shipboard flow field on helicopter operating when landing[J]. Transaction of Nanjing Aeronautical and Astronautical University, 1999 (31), 6: 614-619.

[3]. WANG Shi-cun. Helicopter Aerodynamics[M], Aviation Books Association. 1985: 135-140.

[4]. Gaonkar G H, Peters D A, Review of Dynamic Inflow Modeling for Rotorcraft Flight Dynamics[J]. Vertica, $1988,12(3): 213-242$.

[5]. Cheeseman I C, Bennett W E , The Effect of the Ground on a Helicopter Rotor in Forward Flight[R]. ARCR\&M. 1955, 3021

[6]. Hong Zhang, etc. A Simulation Model of Ship Ground Effect for Rotorcraft / Ship Interaction Study[C], American Helicopter Society 51st Annual Form, 1995. 